\title{
Logical implications for regulatory relations represented by verbs in biomedical texts
}

\author{
Sine Zambach ${ }^{1}$ \\ ${ }^{1}$ Roskilde University, Roskilde, Denmark
}

\begin{abstract}
Relations used in biomedical ontologies can be very general or very specific in respect to the domain. However some relations are used widely in for example regulatory networks. This work focuses on positive and negative regulatory relations, in particular their usage expressed as verbs in different biomedical genres and the properties of the relations.
\end{abstract}

\section{Introduction}

In the research area of biomedical ontologies, the work with formal relations has recently reached a level where integration in a larger project is possible ${ }^{1}$. Using a thorough analysis of the actual logic implications of the relations has been suggested ${ }^{2}$.

With the opportunity of using new DL-formalism $\mathcal{E L}+$ and reasoning tool ${ }^{3}$, CEL, relations can be treated as modules with complex inclusions themselves.

This study is concerned with the two relations, positive and negative regulation relations. The relations have been investigated in corpora and in relation to their logical implications. For an easy reading we call them stimulates and inhibits.

\section{Results}

Inhibit and stimulate are relations that - in a biochemical pathway actually contains a special kind of inheritance, e.g. if $x$ inhibits $y$ and $y$ stimulates $z$, then you can deduce that $x$ stimulates $z$ as formulated in FOL using stim for stimulation and inh for inhibition:

$$
\begin{aligned}
& \forall x(A(x) \rightarrow \forall y(B(y) \wedge \operatorname{stim}(x, y) \wedge \forall z(C(z) \wedge \operatorname{stim}(y, z) \rightarrow \operatorname{stim}(x, z))) \\
& \forall x(A(x) \rightarrow \forall y(B(y) \wedge \operatorname{inh}(x, y) \wedge \forall z(C(z) \wedge \operatorname{inh}(y, z) \rightarrow \operatorname{stim}(x, z))) \\
& \forall x(A(x) \rightarrow \forall y(B(y) \wedge \operatorname{inh}(x, y) \wedge \forall z(C(z) \wedge \operatorname{stim}(y, z) \rightarrow \operatorname{inh}(x, z))) \\
& \forall x(A(x) \rightarrow \forall y(B(y) \wedge \operatorname{stim}(x, y) \wedge \forall z(C(z) \wedge i n h(y, z) \rightarrow \operatorname{inh}(x, z)))
\end{aligned}
$$

These properties can also be expressed in the $\mathcal{E L}+$ language and are called complex role inclusions: inhibit o stimulate $\subseteq$ inhibit etc and the new CELmodule can handle axioms like this.

The verb frequencies in biomedical corpora are ranked in figure 1 . This experiment reveals that verbs representing the regulatory relations has a special use in for example Medline abstracts and biomedical patents compared to the British National Corpus (BNC). Whereas the 10 most frequent verbs from BNC has a low rank in all corpora, the regulatory relations seems to be more specific for the biomedical texts.

\section{Conclusion}

Corpora analysis indicates that the words representing positive and negative processes have an important role in biomedical texts which should be investigated further. In addition to this, an implementation in DL is suggested such that these relations can be expressed in a way that facilitates e.g. reasoning. Files can be found at: ruc.dk/ sz/ICBO09/relations.

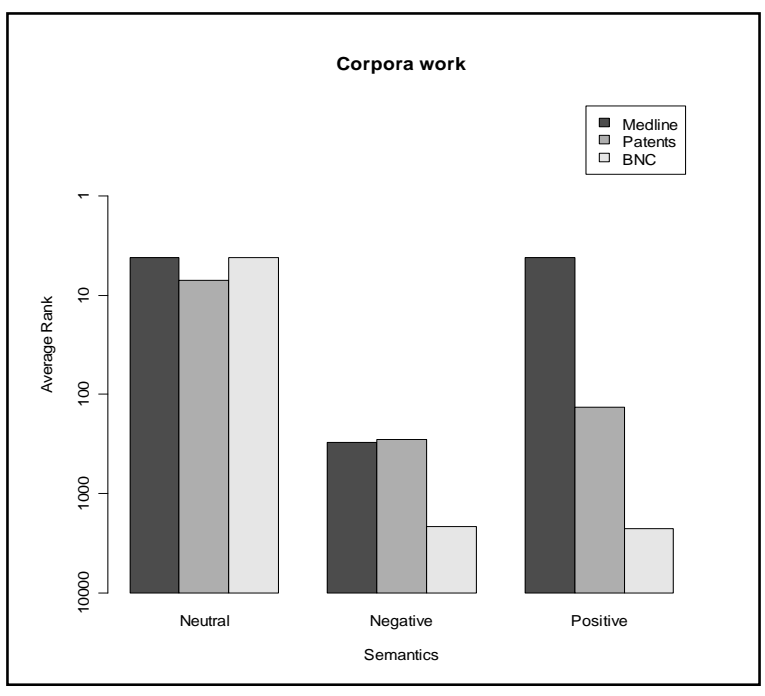

Figure 1. Average rank of regulatory relations for biomedical patents, Medline abstracts and BNC.

\section{References}

1. Smith, B. et al., Relations in biomedical ontologies. Genome Biology 2005, 6:R46.

2. Smith, B. \& Rosse, C, The role of foundational relations in the alignment of biomedical ontologies, Medinfo. 2004, 444-448.

3. Baader, F., Lutz, C., Suntisrivaraporn, B., CEL a polynomial-time reasoner for life science 
ontologies. Lecture Notes for Computer Science, Springer Berlin / Heidelberg, 2006, 287-291. 